\title{
Review on fire explosion research of crude oil storage tank
}

\author{
Longfei Li ${ }^{1, a^{*}}$, Longyu Dai ${ }^{2, b}$ \\ ${ }^{1}$ Department of Oil and Gas Storage and Transportation Engineering, China University of Petroleum-Beijing at Karamay, Karamay, Xin- \\ jiang Uygur Autonomous Region, China. \\ ${ }^{2}$ Department of Oil and Gas Storage and Transportation Engineering, China University of Petroleum-Beijing at Karamay, Karamay, Xin- \\ jiang Uygur Autonomous Region, China.
}

\begin{abstract}
With the rapid development of the world economy, the petrochemical industry energy reserve strategy and production demand increase, petrochemical storage tank farm scale is also expanding, and continue to large, intensive direction of development. In recent years, there are many disastrous accidents such as oil leakage, explosion and combustion of storage tank, so it is necessary to study the combustion and explosion behavior of storage tank. This paper studies the mechanism of explosion combustion in crude oil tank area, summarizes and states the progress of crude oil explosion combustion research at home and abroad, and lists several common risk evaluation methods for tank risk management, which can provide technical support for safety management and accident emergency rescue through qualitative, quantitative evaluation and accident consequence simulation calculation. Therefore, we should sum up the experience and lessons from the accident, strengthen the theoretical research, to avoid the recurrence of similar accidents, and effectively guarantee the safety of national energy reserves.
\end{abstract}

\section{Introduction}

\subsection{Significance of risk management in crude oil storage}

With the impact of the oil crisis and the war between the oil exporting countries (such as the Gulf War), the expansion of strategic petroleum reserve has become a subject of worldwide concern. The improvement of the petroleum reserve system plays a decisive role in ensuring national energy security, and countries also further accelerate the construction of national petroleum strategic reserve. America's and Japan's national strategic oil reserves already provide more than 200 days' supply. China is the world's second largest oil consumer, with an external dependence of over $60 \%{ }^{[1]}$. In order to ensure energy security, China is striving to build and expand its oil reserve capacity and accelerate the pace of building a national oil strategic reserve.

At present, China has nearly 7,000 large storage tanks, with a single tank capacity of up to 200,000 square meters. But in recent years, there have been many tank accidents in China, which have caused heavy casualties and property losses. There are many reasons for the frequent accidents. On the one hand, because most of China's oil reserves are located along the Yangtze River, in case of serious accidents such as fire and explosion, it will seriously harm the ecological environment and public safety. On the other hand, with the development of petroleum economy, China's tank areas show the characteristics of "three difficulties" : single tank and large storage capacity - difficult fire; Large amount of oil in and out, complicated process -difficult to manage; Mixing of petroleum and hazardous chemicals in tank areas -- Difficult to control public safety. Therefore, how to avoid fire, explosion and other accidents to minimize casualties and property losses is of great significance to the risk management of crude oil storage.

\subsection{Research significance of explosion and combustion of crude oil storage tank}

Crude oil tank farm is an important place for crude oil storage and transportation. It contains a large number of storage tanks, large capacity tanks, many auxiliary facilities and complicated technology, which is the hazard source of fire and explosion. Tank area is also where management is weak at the same time, crude oil is mostly flammable and explosive, poisonous and harmful, if the tank to produce larger defects, such as the crack extension, severe corrosion, structural instability, etc.), cause the medium leakage, is easy to cause the disastrous accidents such as fire, explosion, a serious threat to people's life and property safety.

Due to technical and management reasons, a large number of domestic storage tanks are in overdue or long-term continuous operation state, and lack of effective testing means and scientific risk assessment methods, their safety state is poor. In recent years, tank leakage, fire and explosion accidents occur frequently, which has caused serious social impact. Such as

*Corresponding author: ${ }^{\mathrm{a}} 15038455757 @ 163 . c o m$

b1009699462@qq.com 
huangdao oil depot "8.12" serious fire accidents, oil tank explosion fire of 23000, the top of the tank was ignited the leakage of oil and gas layer, has ignited the surrounding tank, causing a direct economic loss of 35 million yuan ${ }^{[2]}$, and as on November 22, 2013, sinopec east yellow major oil pipeline leak explosion accident, the accident killed 64 people and 145 people were injured, in December 2005, the British Buncefield tank explosion, $300 \mathrm{t}$ oil overflow tank, caused by fire duration more than $60 \mathrm{~h}$, burned more than 20 large volume oil tank, The direct economic loss is about 3.5 billion yuan. On September 6, 2001, a fire occurred in beishan Crude oil tank farm of Maoming Petrochemical Company, and on July 12, 2009, 5.4 oil tank explosion occurred in Taizhou city, Jiangsu Province, etal. In order to ensure the safety of production and people's property, it is urgent to analyze and study the risks of tank farm.

\section{Types and causes of tank fire and explosion accidents}

\subsection{Cause of tank fire accident}

Shen Guoguang etal. ${ }^{[4]}$ conducted a large number of investigations on the causes of fire accidents in domestic storage tanks, and found that fires caused by open fire accounted for $52.4 \%$ of the total accidents, ranking the first. In addition, lightning and static electricity accounted for about $10 \%$, which is also an important cause of fire; Other external causes, such as improper operation by staff and the occurrence of sparks, account for about $30 \%$ of the total accidents.

According to the large-scale atmospheric tank fire study jointly conducted by 16 oil companies including BP and Shell ${ }^{[5]}$, there are 15 20 large oil tank fire accidents every year. Three common types of tank fire accidents including floating roof tank, fixed roof tank and external floating roof tank were counted, including 33 domestic cases and 38 foreign cases. The accident analysis and statistics show that the proportion of fire accident of external floating roof tank is $35 \%$.

\subsection{Types of fire accidents}

Zhao Jinlong and Huang Hong etal. ${ }^{[6]}$ counted 88 cases of external floating roof tank accidents at home and abroad, founded that seal fire is the most common type of fire, followed by full-area fire and fire embankment fire. The following is a brief description and analysis of the above fire types.

\subsubsection{Seal ring fire}

Seal ring fire: The mixture of oil and gas in the explosion range of the sealing device burns or even explodes when it meets the ignition source. It is a common type of tank fire.

Sealing ring burning area is small, if can timely control the fire, generally will not develop into a full area fire and fire pool fire. However, if the fire is not contained, the fire can last for several days. In addition, the high temperature emitted by the combustion will cause intense heat radiation, leading to the damage of the floating roof and further development of the floating roof pool fire. Once the tank wall is damaged, the oil will leak out of the tank and form a fire pool. In the process of sealing ring fire extinguishment, in order to avoid the floating roof sinking into the tank due to excessive weight and causing full-area fire, it is necessary to control the amount of fire water and self-spraying system of storage tank to the floating roof, improve the success rate of fighting and control the spread of fire, which are the key factors to avoid the expansion of fire influence ${ }^{[7]}$.

\subsubsection{Fire in the pool}

Fire in floating roof pool and fire dike pool: in the floating roof and fire dike pool formed due to oil leakage, open fire is triggered. Baham etal. ${ }^{[8]}$ showed that about $80 \%$ of domino accidents caused by fire were the result of pool fires. For large floating roof tanks, pool fire in the fire embankment is one of the main fire types ${ }^{[9]}$. Fire pool fire is relatively easy to put out, to put out the floating roof pool fire need to avoid excessive fire water will sink the float plate, into a full area fire. If the pool fire is not properly controlled and spreads to surrounding storage tanks, a group tank fire may occur.

\subsubsection{Full area fire}

After the floating plate is seriously tilted or sunk, most of the oil surface of the floating roof tank is directly exposed to the atmosphere, and the volatile amount of oil and gas increases sharply. After mixing with the air, a large amount of explosive oil and gas is formed, which is easy to cause full-area fire in the filling roof when it meets the ignition source.

\subsection{Type of explosion accident}

In recent years, there have been many leakage accidents of gas pipelines and storage tanks in China, which may lead to fire or explosion accidents if the leakage is serious. Explosion of other fire dikes pool fire full area fire sealing ring fire explosion accidents can be divided into explosion in tank and explosion outside tank:

Tank explosion: When cleaning the tank, high concentration of oil vapor remains in the tank, air enters the tank and mixes, reaches the explosion limit and mixes, reaches the explosion limit and ignites with open flame, steam cloud explosion will occur. Explosion outside tank: When a tank leaks, if not detected in time, the leaked oil evaporates in the air. When the concentration of oil vapor reaches the explosive limit, it is exposed to an open flame, and steam cloud explosion occurs. 


\section{Research progress of tank fire explosion at home and abroad}

In the process of development, storage and transportation of crude oil, the accidents of combustion and explosion of crude oil pipelines and storage tanks occur many times. Once the combustion and explosion of basic loaded storage tanks for crude oil occurs, it will be a big challenge to extinguish and maintain later. Therefore, studying the mechanism of combustion and explosion in crude oil storage tanks, finding out the law of explosion derivation and development, and proposing reasonable prevention and intervention measures will be beneficial to reduce the occurrence of combustion and explosion accidents based on the above research results.

At present, the main research methods for oil and gas leakage, combustion and explosion are: mathematical model and numerical simulation. The mathematical model research methods mainly include gas leakage mathematical model, jet fire mathematical model, gas cloud explosion mathematical model and so on. With the progress of information technology, researchers at home and abroad use computer simulation of high processing power to simulate the process of the explosion. The numerical simulation method has the advantages of economy, safety and strong anti-interference, but its disadvantage is that the simulation results must be verified by experimental data or mathematical method. The combination of mathematical model and numerical simulation can fully understand and discover the explosion law ${ }^{[10]}$.

\subsection{Domestic research status}

Chen Shiwei et al. ${ }^{[11]}$ established a simulation experiment system for oil and gas explosion in storage tank with No. 90 gasoline as the experimental working medium, and on the basis of the simulation experiment, respectively studied the influence of initial oil and gas concentration and initial temperature on the explosion pressure. The experimental results show that there is a critical concentration of oil and gas mixture at the time of explosion, the critical oil and gas concentration is $2.5 \%$, and the critical initial temperature is $306 \mathrm{~K}$. Under the critical value, the explosion pressure of oil and gas is the maximum., the explosion pressure increases with the increase of oil and gas concentration, and the flame propagation speed increases rapidly. When the concentration of oil and gas is greater than the critical concentration, and when the concentration is greater than the critical concentration, the explosion pressure decreases with the increase of the concentration of oil and gas and the flame propagation velocity decreases.

Using acetylene/air mixture as experimental medium, wang wei etal. ${ }^{[12,13]}$ established a scale model of floating roof type storage tank of 150,000 m3, 100,000 $\mathrm{m} 3$ and $50,000 \mathrm{~m} 3$. The impact experiment of the model shows that the roof area of the floating roof tank is the weak part of the whole structure, and the plastic deformation is easy to occur under the impact of ex- plosion. The experimental results show that the shock wave from the high temperature steam cloud caused by acetylene explosion is the main factor causing the tank wall damage.

Zhu Baikang etal. ${ }^{[14]}$ established a laboratory model of floating roof tank, studied the distribution of oil and gas in the primary and secondary sealing space of floating roof tank under different environments, and used the sadovsky explosion shock wave peak overpressure formula to build the explosion pressure distribution model. The experimental results show that the wind speed and direction affect the distribution of oil and gas in the sealing ring of the floating roof tank, and the wind pressure aggravates the damage effect of the explosion shock wave on the tank wall. It is concluded that the explosion and combustion accident can be avoided by improving the ability of a seal to withstand pressure waves and avoid being damaged by pressure waves.

Jiang Xinsheng, Wei Shuwang etal. ${ }^{[15]}$ set up an oil and gas explosion experiment system for long and narrow pipeline with gasoline as the experimental medium, and conducted an oil and gas explosion experiment. The maximum explosion overpressure, boost rate, flame intensity and flame duration of oil and gas in the pipeline with the oil and gas volume fraction concentration ranging from $1.25 \%$ to $2.65 \%$ were experimentally studied. The results show that with the increase of the initial oil/gas volume fraction, the maximum explosion overpressure value and boost rate along the pipeline show a trend of first increasing and then decreasing, while the flame duration is first decreasing and then increasing, and it is the shortest when the oil/gas volume fraction is $1.75 \%$.

$\mathrm{Yu}$ Bo ${ }^{[16]}$ simulated the process of gas cloud explosion, discussed the distribution law of pressure field and impulse field of gas cloud explosion, analyzed the dynamic response and failure characteristics of concrete structure under gas cloud explosion, and discussed the influence law of gas cloud size, ignition energy, ignition position and other factors on the deflagration strength of gas cloud.

Using gasoline-air as the experimental medium, wang bo etal. ${ }^{[17,18]}$ studied the oscillation propagation characteristics of gasoline-air mixed gas explosion in a slender closed pipe, and conducted explosion experiments in pipes with different aspect ratios (round, square and spherical). It is found that the oscillating explosion of oil and gas occurs only within the oscillating explosion limit, and the pressure of oil and gas explosion is related to the concentration of oil and gas and the length-diameter ratio of the pipeline. With the increase of the length-diameter ratio of the pipeline, the range of the explosion limit increases gradually, and the proportion of the oscillation explosion limit in the oil-gas explosion limit increases gradually. With the increase of oil and gas concentration, the maximum explosion pressure first increases and then decreases. With the increase of oil and gas concentration, the time when the maximum explosion pressure reaches the peak shows a trend of decreasing first and then increasing. 
Zheng Lichong ${ }^{[19]}$ simulated the steam cloud explosion inside the steel storage tank, calculated the overpressure time history of each point in the tank, and divided the tank into several equal load areas according to the characteristics of overpressure distribution change, providing a simplified explosion load model. Based on johnson-Cook material constitutional model parameters and explosion load simulation results, LS-DYNA was used to numerically simulate the explosion response of steel storage tanks, and the structural dynamic response and failure modes of steel storage tanks with different sizes and different roof forms under internal explosion were analyzed.

Wang Shimao etal. ${ }^{[20]}$ using computer software through the adoption of the total energy of $\mathrm{k}$ - epsilon turbulence model and simplified the oil and gas combustion of two step reaction tank was simulated under the condition of opening at the top of the tank explosion law of 90 \# gasoline, a numerical calculation model to verify the results of numerical simulation, get in different period of oil and gas explosion pressure change process with time, the analysis of the error of the numerical calculation model and numerical simulation.

Taking gasoline as the research object, Qian Haibing etal. ${ }^{[21]}$ studied the explosion limit of oil and gas explosion in a closed tank under experimental conditions, and found that the explosion limit of oil and gas in a closed space was $1.5 \% \sim 4.6 \%$, and when the volume fraction of oil and gas was about $2.5 \%$, the combustion explosion intensity of oil and gas in a closed tank was the largest.

For under explosion shock loading tank structure coupling dynamic response and failure mechanism of YangDu ${ }^{[22,23]}$ FEM is proposed - SPH method of fluid and structure coupling, deduces a kind of structure associated with strain rate failure criterion, pressure vessel and pipe blends was studied the influence of flammable gas explosion consequences, and verify the dynamic fracture characteristics of the material.

Du Yang, Yang xiaofeng etal. ${ }^{[24,25]}$ took gasoline as the research object, studied the turbulent combustion explosion model based on various mechanisms, and established the two-step reaction of combustion reaction, with different mechanism and different energy released in each step. The coupling mechanism between flame and pressure wave has been correctly and reasonably explained by experiments of oil and gas combustion explosion and numerical simulation in narrow space.

Zhang Peili etal. ${ }^{[26]}$ adopted an assembled shock tube device with a length of $5 \mathrm{~m}$ and a diameter of $0.2 \mathrm{~m}$. By adding nitrogen to the oil and gas, they found that nitrogen can inhibit the occurrence of oil and gas explosion, and successfully obtained the process of attenuation and extinction of oil and gas under the action of nitrogen suppression.

Du Yang song-lin wu, etal. ${ }^{[27]}$ to study the combustion of circular pipe explosion pipeline in a sealed space, puckering open drain blasting experiment and double hole open drain blasting experiment, through the visual analysis found that gas jet and flame open drain blasting process is divided into what shall the mushroom cloud formation, gas injection and what shall continue jet flame speed and the flame is extinguished four process.

When analyzing the dynamic response of the spherical tank under the action of external explosive load, B.Y. Zhang ${ }^{[28]}$ simulated the vapor cloud explosion of the tank by the TNT equivalent method, and studied the pressure distribution and dynamic response of the spherical tank wall surface with the tank capacity of 1000 cubic meters.

Sun Enji etal. ${ }^{[29]}$ calculated the quality of liquid ammonia leakage based on realizable K-Epsilon finite element numerical simulation analysis method in view of the leakage accident of liquid ammonia storage tank, and simulated and studied the diffusion law of ammonia gas in the air at different tank leakage locations and leakage amounts. The results show that the location of the outlet has little influence on ammonia diffusion, while the location of the outlet has great influence on ammonia diffusion.

Zong Hui ${ }^{[30]}$ studied the failure probability of tank unit fire and explosion accidents based on the accident-tree analytic hierarchy process, and established a complex network accident propagation model based on Markov chain on the basis of domino effect, explored the interaction between each tank unit in the tank area, and solved the final failure probability. According to the principle of grid division technology and risk superposition, the risk distribution of tank farm was calculated and the risk distribution map was drawn considering the population distribution of tank farm and nearby areas.

Mark chan ${ }^{[31]}$ and who uses FDS simulation software, ANSYS, was studied in three straight line configuration for the volume of 5000 cubic meters of diesel dome-roof tank, two cans of the combustion characteristic of the whole area of fire, thermal radiation intensity distribution of tank are analyzed, and further studied the two pool fire storage tank under the action of thermal response characteristics.

\subsection{Research status abroad}

A. Velikorodny ${ }^{[32]}$ proposed a new premixed combustion model and improved it by introducing the estimation of the transient integral length scale coupled with the velocity gradient field. This model can be used to verify the results of numerical calculations (over a wide range and in different combustion states). The paper also presents a method to verify the combustion model and its possible improvement to the non-uniform mixtures in space.

Olavr. Hansen ${ }^{[33]}$ established BLEVE model with CFD model and verified the model of explosion wave.

Josef Hasslberger ${ }^{[34]}$ proposed an efficient CFD model for simulating hydro-air explosion (including transition to detonation) by revising the Weller deflagration model, which was verified by indoor DDT experiment.

Through the model calculation, the pressure load and the reflection of explosion wave changing with 
time are obtained. Jingde Li etal. ${ }^{[35]}$ studied the effect of separation gap on gas explosion pressure in and around the tank, and applied the research method of numerical simulation (CFD) and experimental mutual verification. A series of gas explosion arrangements with different gas intervals were studied experimentally. By CFD simulation, the gas explosion under different gas cloud cover was considered, and the influence of the separation gap at different positions on the explosion pressure of storage tank was studied.

Olavr. Hansen etal. ${ }^{[36,37]}$ studied the shape and duration of explosive loads and the optimization methods for extracting loads from different types of targets by using the method of fluid dynamics (CFD) numerical simulation combined with experiments. Experiment in the choice of drag coefficient $\mathrm{Cd}$ is discussed, and simulation of the full scale explosion experiment, further proves the FABIGTN - 08 the rationality of the pressure distribution function is introduced, and put forward for pipe explosion intensity bigger, should adopt conservative drag coefficient $\mathrm{Cd}$, and put forward to simulate experiment, in order to correctly estimate the load from the simulated data, it is necessary to drag coefficient $\mathrm{Cd}=2.0$.

Purvali Chaudhari etal. ${ }^{[38]}$ used machine learning algorithm, random forest (RF), artificial neural network (ANN), and non-machine learning algorithm -- Genetic function approximation (GFA) to establish a quantitative structure-property relationship (QSPR) regression model for the minimum ignition energy (MIE) prediction of 60 flammable compounds.

Derek Bradley, Philiph. Gaskell etal. ${ }^{[39]}$ established an experimental database of jet flame heights and flame lift distances covering six fuels and different flow states. The computer simulation of turbulent jet flame shows that for a given mixture, the turbulent combustion rate increases the volume of the reaction mixture mainly in a near proportional manner.

Reinders etal. ${ }^{[40]}$ took LPG tanks as the research object and proposed a tank heat balance model based on experiments, which was used to predict the pressure and temperature variation law of multi-layer adiabatic tanks containing compressed liquefied gases after heating. The results show that when the thermal conductivity of the insulation layer is constant, the variation law of the model pressure and temperature with time is in good agreement with the experimental results.

Pantousa ${ }^{[41]}$ fixed roof tank was studied by finite element numerical solution method in the thermal buckling behavior of pool fire scenario, consider the tank size, tank spacing number, fuel, wind speed, burning pot on the thermal buckling behavior response of storage tank, but involves only considered part of the pool fire burning tank number influence on the critical buckling temperature and failure time, not of pool fire coupling mechanism further explored.

Wan etal. ${ }^{[42,43]}$ proposed a two-pool fire cuboid flame model and a weighted multi-point source model on the basis of the piecewise function established to predict the flame temperature, and studied the emis- sivity of the flame, the average flame temperature of the model and the corresponding blackbody thermal radiation power.

\subsection{Safety management evaluation of oil depot}

Since the supply of oil depot for national petroleum storage and has important strategic value, and due to store flammable and explosive properties of medium, not prevent oil depot fire and explosion accident, need to adopt a scientific and reasonable analysis methods for oil depot or tank farm of risk assessment, according to the size of accident risk and possible accident consequences, safety management and puts forward corresponding preventive measures ${ }^{[44]}$.

The commonly used safety evaluation methods include qualitative evaluation method, quantitative evaluation method, semi-quantitative evaluation method, scene simulation evaluation method, etc. The qualitative evaluation methods include risk matrix method, safety checklist method (SCL), HAZOP analysis method, fault tree analysis method (FTA), etc. The quantitative evaluation methods include probabilistic evaluation (PRA), risk index, etc.

Semi-quantitative evaluation methods include index evaluation, etc. ${ }^{[45]}$. According to the specific situation of oil depot or tank farm, quantitative and qualitative evaluation methods can be adopted comprehensively. The results of qualitative and quantitative evaluation and simulation calculation of accident consequences can provide technical support for safety management and emergency rescue of accidents.

\section{4 summary}

Firstly, this paper introduces the significance of risk management in crude oil tank area. Since most crude oil is inflammable and explosive, if there are large defects in storage tanks, the leakage of crude oil media will easily lead to catastrophic accidents such as fire and explosion. By studying the mechanism of explosive combustion of crude oil tank, this paper summarizes and states the research progress of explosive combustion of crude oil at home and abroad, and lists several commonly used risk assessment methods for the risk management of oil tank.

Floating roof tank fire is the most common type of tank fire. Common fire types of floating roof tank are seal fire, full area fire and fire dike fire. Sealing ring fire is the most common type among several fire types. Most of the researches on tank fire at home and abroad focus on numerical simulation and empirical model. The commonly used empirical models are: gas leakage mathematical model, jet fire mathematical model, gas cloud explosion mathematical model.

With the progress of science and technology, the numerical simulation method develops rapidly. The numerical simulation method is widely used by scholars at home and abroad because of its advantages of economy, safety and strong anti-interference. For ex- 
ample, turbulence model analysis method based on Realizable K-Epsilon, fluid dynamics (CFD) model, FLUENT finite element numerical solution method, etc. In addition, the research method of mutual verification of mathematical model and numerical simulation can enable people to fully understand and find the law of explosive combustion, so that the research results can be better applied to the actual production.

The large-scale intensive development of crude oil storage tanks and storage tanks not only serves the development of national crude oil reserves better, but also leads to many disastrous accident consequences. Under this background, safety management evaluation and consequence prediction of major accidents are particularly important. At the end of this paper, several common safety evaluation methods are summarized. The results of qualitative and quantitative evaluation and simulation calculation of accident consequences can provide technical support for safety management and emergency rescue.

\section{Reference}

1. Li J, WU J.G. (2016) Countermeasures for China's Petroleum Reserve Construction. International Petroleum Economics, 24(12): 34-37+56.

2. Zan Q, Jiang T. (1999) A Ten-year Review of the Fire accident of Huangdao Oil Depot on August 12. Chemical Labor Protection, 20 (6) :204-207.

3. HERBERT I. (2010) The UK Buncefield incident-The view from a UK risk assessment engineer. Journal of Loss Prevention in the Process Industries, (23): 913-920.

4. Shen, G.B. (2020) A Study on the Causes and Sustainability of The U.S. Trade Deficit --A Review of the Study on the U.S. Trade Deficit. Journal of Changsha University of Science and Technology (Social Science Edition),, 35(06): 105-110.

5. Zhang Q.L. (2014) Typical Fire cases of Oil storage tanks at home and abroad. Tianjin University Press, Tianjin.

6. Zhao J.L, Tang Q, Huang H, etal. (2015) Quantitative risk assessment of large external floating roof tank area based on numerical simulation. Journal of tsinghua university (natural science edition), 55(10) : 1143-1149.

7. Xie F, Luo Y.P, Zhang Y.L. (2017) Analysis of oil and gas leakage and Diffusion of sealing Ring of large Floating roof tank. Fire Science and Technology, 36(10): 1458-1460.

8. Bahman Abdolhamidzadeh, Tasneem Abbasi, D. Rashtchian, etal. (2010) Domino effect in process-industry accidents - An inventory of past events and identification of some patterns. 24(5): 575-593.

9. Zhang D. (2004) Applied Research on environmental Risk Assessment of large oil tank Spill fire accident. Capital University of Economics and
Business.

10. Qiu Y.Q. (2017) Study on the Mechanism of oil and gas explosion and combustion in large crude oil storage Tanks. Zhejiang Ocean University.

11. Chen S.W., Du Y, Wang B. (2007) Study on the explosion law of oil and Gas in oil tanks. Journal of Safety and Environment, (03): 102-104.

12. Lu S.Z., Wang W, Zhang B.Y. (2011) Experiment on explosion failure mechanism of large Floating roof storage Tank. Explosions and Shocks, 31(02): 158-164.

13. Lu S.Z., Wang W, Chen W.D.(2005) Explosion impact failure of floating roof storage Tank. Explosion and Impact, 35(05): 696-702.

14. Zhu B.K., Ding B., Wen J.J. (2015) Simulation of oil and gas distribution and explosion pressure in the primary and secondary sealing space of floating roof tank. Safety and environmental engineering, 22(01): 148-151.

15. Jiang X.S., Wei S.W., Yuan G.Q., etal. (2016) Distribution characteristics and analysis of oil and gas explosion flow field in narrow and narrow pipelines. China safety production science and technology, 12(08): 130-134.

16. Yu B. (2015) Numerical Simulation and structural response Analysis of gas cloud Explosion field. Nanjing University of Science and Technology.

17. Wang B., Du Y., Li G.Q., etal. (2017) Study on the explosion characteristics of oil and gas in slender closed pipe. Vibration and Impact, 332(4): 432-438.

18. Wang B. Du Y., Qi S., etal. (2017) Oscillation propagation characteristics of oil and gas explosion in long and narrow closed pipe. Vibration and Impact, 36(17): 97-103.

19. Zheng L.C. (2015) Numerical Simulation of explosive dynamic response and thermal buckling of large steel storage tanks. Zhejiang University.

20. Wang S.M., Du Y., Zhang S.B., etal. (2015) Numerical Simulation of oil and Gas explosion in tank under top opening condition. Journal of Logistics Engineering College, 31(04): 51-56.

21. Qian H.B., Du Y., Ye Z.B. (2005) (in Chinese) Experimental study on the initial fire model of oil storage tank. Journal of The College of Logistics Engineering, 21(3): 7-9.

22. Yang D., Fan Z., Li M., etal.(2019) Consequence analysis of premixed flammable gas explosion occurring in pipe using a coupled fluid-structure-fracture approach. Journal of Loss Prevention in the Process Industries, 57: 81-93.

23. Du Y., Zhang F, Zhang A, etal. (2016) Consequences assessment of explosions in pipes using coupled FEM-SPH method. Journal of Loss Prevention in the Process Industries, 43: 549-558.

24. Du Y., Yang X.F., Jiang X.S., etal. (2006) Study on turbulent explosive combustion model based on 
multiple control mechanisms. Journal of Engineering Thermophysics, 27(6): 1045-1047.

25. Du Y, Yang X F, Jiang X S, etal. (2006) Turbulence explosion combustion model study based multicontrol mechanisms. Journal of Engineering Thermophysics.

26. Zhang P.L., Du Y. (2016) Nitrogen non-premix inhibition experiment of oil-gas explosion. Explosion and Impac, 36(3): 347-352.

27. Wu S.L., Du Y, OU Y.H. etal. (2016) Experimental study on side-by-side oil and gas leakage of cylindrical pipeline. Explosion and Impact, 36(5): 680-687.

28. Zhang B.Y., Li H.H., Wang W. (2015) Numerical study of dynamic response and failure analysis of spherical storage tanks under external blast loading. Journal of Loss Prevention in the Process Industries, 34: 209-217.

29. Sun Y.J., Li H.G., Wang M. (2017) Numerical simulation of ammonia leakage based on Realizable K - turbulence model. China Work Safety Science and Technology, 13(02): 114-118.

30.Zong H. (2016) (in Chinese) Research on failure Probability and Risk Evaluation of tank group in tank Farm. Southeast University.

31. Chen G.H., Zhang X.Y., Zhou Z.H., Zeng T. (2020) Numerical simulation of thermal response of diesel dome tank under two pool fire coupling.Chemical Engineering Progress, 39(11): 4342-4350.

32. Velikorodny A., Studer E., Kudriakov S., etal. (2015) Combustion modeling in large scale volumes using EUROPLEXUS code. Journal of Loss Prevention in the Process Industries, 35: 104-116.

33. Hansen O.R., Kjellander M.T. (2016) CFD modelling of blast waves from BLEVEs. Chemical Engineering Transactions, 48:199-204.

34. Hasslberger J., Boeck L.R., Sattelmayer T. (2015) Numerical simulation of deflagration-to-detonation transition in large confined volumes. Journal of Loss Prevention in the Process Industries, 36: 371-379.

35. Jingde L., Hong H., Shi Y.C., etal. (2018) Experimental and computational Fluid Dynamics study of separation gap effect on gas explosion mitigation for methane storage tanks. Journal of Loss Prevention in the Process Industries, 55: 359-380.

36. Hansen O.R., Kjellander M.T., (2016) Pappas J A. Explosion loading on equipment from CFD simulations. Journal of Loss Prevention in the Process Industries, 44: 601-613.

37. Olav R.H., Malte T.K., Remi M., etal. (2016) Estimation of explosion loading on small and medium sized equipment from CFD simulations, 41:382-398.

38. Wang B., Zhou L., Xu K., etal. (2016) Prediction of Minimum Ignition Energy from Molecular
Structure Using Quantitative Structure-Property Relationship (QSPR) Models. Industrial \& Engineering Chemistry Research, 56(1).

39. Bradley D., Gaskell P.H. (2016) Jet flame heights, lift-off distances, and mean flame surface density for extensive ranges of fuels and flow rates. Combustion and Flame, 164: 400-409.

40. REINDERS J.E.A., VELTHUIS J.F.M., (2019) SPRUIJT M P N. Pressure and temperature increase of LPG in a thermally coated pressure vessel exposed to fire: experimental and model results. Journal of Loss Prevention in the Process Industrie, 57: 55-60.

41. PANTOUSA D. (2018) Numerical study on thermal buckling of empty thin- walled steel tanks under multiple pool-fire scenarios. Thin-Walled Structures, 131: 577-594.

42. WAN H.X., GAO Z.H., JI J., etal. (2018) Predicting heat fluxes received by horizontal targets from two buoyant turbulent diffusion flames of propane burning in still air. Combustion and Flame, 190: 260-269

43. WAN H.X., GAO Z.H., JI J., etal. (2019) Experimental study on flame radiant heat flux from two heptane storage pools and its application to estimating safety distance. Energy, 182: 11-20.

44. Shuai J. (2006) Safety Evaluation of crude oil depot. Chinese Journal of Safety Science, (10): $120-125+1$.

45. SY/T6276-1997. (1997) Oil and Gas Industry Health, Safety and Environmental Management System. Beijing: China Petroleum Press. 